\title{
Biología ReProductiva y ROBO DE NÉCTAR EN SALVIA GESNERIFLORA (LAMIACEAE) Y SUS CONSECUENCIAS EN EL ÉXITO REPRODUCTIVO
}

\author{
Eduardo Cuevas-García ${ }^{1,3}$, Aaron Alcalá-Guerra ${ }^{1}$, Yunuhen E. Baños-Bravo ${ }^{1}$ \\ y AlejandRo FloRes-PalaCios ${ }^{2}$ \\ ${ }^{1}$ Universidad Michoacana de San Nicolás de Hidalgo, Facultad de Biología, Morelia, Michoacán, México \\ ${ }^{2}$ Centro de Investigación en Biodiversidad y Conservación (CIByC) \\ Universidad Autónoma del Estado de Morelos, Cuernavaca, Morelos, México \\ ${ }^{3}$ Autor para la correspondencia: eduardocuevas5@gmail.com
}

\begin{abstract}
Resumen: Los robadores de néctar perforan flores para tener acceso al néctar desde el exterior de la flor y, en la mayoría de los casos no contribuyen en la polinización. Las flores de Salvia gesneriflora son perforadas por aves e insectos, lo que podría disminuir el éxito reproductivo de esta especie. En este estudio, se evaluó el sistema reproductivo y el efecto del robo de néctar en distintos componentes del éxito reproductivo de S. gesneriflora. En tres poblaciones se protegieron artificialmente las flores del robo. Se encontró que $S$. gesneriflora es una especie autocompatible, pero no presenta autofecundación espontánea, debido a que sus flores presentan hercogamia y dicogamia (separación espacial y temporal de las funciones sexuales). Las flores autofecundadas manualmente produjeron $75 \%$ menos semillas que las fecundadas por exocruza. Aunque el robo de néctar no afectó la proporción de frutos producidos ni la producción de semillas, sí disminuyó en un $29 \%$ el peso de las semillas y en un $31 \%$ la tasa de germinación.
\end{abstract}

Palabras clave: daño floral, éxito reproductivo, polinización, robadores de néctar, sistema reproductivo.

\begin{abstract}
Nectar robbers perforate generally tubular flowers to access nectar. The flowers of Salvia gesneriflora are robbed by birds and insects that might decrease the reproductive success of this plant. In this study we evaluated several aspects of the reproductive biology of $S$. gesneriflora and the effect of nectar robbing on different components of female reproductive success. In three populations, flowers were protected from nectar robbers. We found that $S$. gesneriflora is a self-compatible species, although autonomous selfing did not occur, probably because their flowers showed herkogamy and dichogamy. Manual self-pollinated flowers produced $75 \%$ fewer seeds than cross-pollinated flowers. Nectar robbing mainly affected seed mass (29\%) and germination rate $(31 \%)$, while fruit set and seed number were not significantly different between robbed and unrobbed flowers.
\end{abstract}

Key words: floral damage, nectar robbers, pollination, reproductive success, reproductive system.

$\mathbf{L}$ a polinización es considerada una interacción mutualista en donde las plantas se benefician con la fertilización de sus óvulos y los animales obtienen recompensas en forma de polen y néctar (Real, 1983). El néctar es una de las principales recompensas que ofrecen las plantas y representa la principal fuente de energía para polinizadores, como los colibríes, debido a la gran cantidad de calorías que proporcionan sus azúcares (Ornelas et al., 2007; Heil, 2011). No obstante, hay insectos y aves que perforan las corolas de las flores o rompen partes de la flor para extraer el néctar desde el exterior, a esas especies se les conoce como robadores de néctar (Inouye, 1980). Los robadores de néctar pueden disminuir las visitas de los polinizadores, en detrimento de la producción de frutos y semillas (Irwin y Brody, 1998; Irwin, 2000). Estudios recientes han encontrado que el robo de néctar puede tener también efectos neutros, e incluso positivos en la producción de frutos y semillas (Maloof e Inouye, 2000; Irwin et al., 2001, Irwin et al., 2010).

Las distintas consecuencias del robo de néctar en la adecuación de la planta dependen principalmente del sistema reproductivo de la especie en cuestión (Zhang et al., 2009). 
En especies autocompatibles, los robadores pueden contribuir a la autopolinzación mientras roban el néctar (Navarro, 2000), y en especies predominantemente de exocruza, el robo de néctar puede afectar el éxito reproductivo (Zhang et $a l ., 2009)$. A pesar de la generalidad del fenómeno del robo de néctar y del impacto que puede tener en la adecuación de las plantas, no ha sido, sino hasta las últimas décadas, que se han incrementado los estudios sobre este fenómeno (Irwin et al., 2010).

Salvia gesneriflora Lindl. \& Paxton es una especie con flores polinizadas por colibríes, cuyas flores son robadas por aves, abejorros y abejas carpinteras (Xylocopa spp.). En este trabajo se estudió experimentalmente aspectos básicos del sistema reproductivo y su relación con el robo de néctar. Se partió de la hipótesis de que si S. gesneriflora es una especie principalmente de exocruza; es decir, que presenta una separación temporal o espacial de las funciones sexuales (dicogamia y hercogamia), lo que evita la autofecundación espontánea y requiere la visita de polinizadores para la producción de semillas; el robo de néctar afectará su éxito reproductivo. Por el contrario, en caso de que $S$. gesneriflora presente fecundación espontánea, el robo de néctar tendrá un efecto neutro o positivo en el éxito reproductivo.

\section{Materiales y métodos}

Sitio de estudio. Este trabajo se llevó a cabo en la región de "Los Azufres", a unos $80 \mathrm{~km}$ al este de Morelia, en el estado de Michoacán, México. El clima predominante es templado subhúmedo (temperatura media anual de $12-18{ }^{\circ} \mathrm{C}$ y precipitación anual de 1,200-1,600 mm; Carranza-González, 1987). El tipo de vegetación corresponde a bosque de pino-encino, siendo Quercus laurina Bonpl., Q. crassipes Bonpl. (Fagaceae), Abies religiosa (Kunth) Schltdl. \& Cham., Pinus pseudostrobus Lindl. y P. teocote Schltdl. \& Cham. (Pinaceae) las especies arbóreas dominantes (Carranza-González, 1987). La vegetación del sotobosque está integrada por especies de los géneros Baccharis, Cestrum, Lupinus y Salvia; y es S. gesneriflora una especie predominante en las poblaciones estudiadas. En esta zona se localizaron tres poblaciones de esta especie en las que se realizó el estudio. Salvia gesneriflora, es un arbusto perenne (0.8-4 $\mathrm{m}$ de altura) endémico del centro de México, que se distribuye desde Jalisco hasta de Puebla (Ramamoorthy, 2005). Las flores son rojas, bilabiadas y tubulares (entre 5-6 cm de largo; Ramamoorthy, 2005), lo que sugiere un síndrome de polinización ornitofílico. Las flores son hermafroditas con dehiscencia diurna y las dos anteras abren simultáneamente (ver resultados). En promedio, cada flor permanece abierta por cinco días (Alcalá-Guerra, 2010), y cada una contiene únicamente cuatro óvulos. Las anteras están cubiertas por el labio superior de la corola y únicamente quedan expuestas cuando los colibríes introducen su pico y activan el mecanismo de palanca (Wester y Claßen-Bockhoff, 2007); mien- tras que el estigma bífido queda expuesto desde que la flor abre. Observaciones preliminares sugieren que los colibríes Eugenes fulgens e Hylocharis leucotis visitan las flores de $S$. gesneriflora introduciendo su pico a lo largo del tubo floral (Baños-Bravo, 2010). Como robadores de néctar, se ha observado al ave Diglossa baritula y a los himenópteros Xylocopa spp. y Bombus ephippiatus. Los tres robadores perforan la base de la flor, generalmente a la altura del cáliz, para extraer el néctar. En el periodo de diciembre de 2009 a abril de 2010, el promedio de flores perforadas a lo largo de la floración en las poblaciones estudiadas fue del 30 a $40 \%$ (Baños-Bravo, 2010).

Biología reproductiva. El estudio de la biología reproductiva únicamente se realizó en la población tres. Se evalúo la presencia de hercogamia en diez plantas seleccionadas al azar y se midió la distancia de las anteras al estigma en cuatro flores por planta. La presencia de dicogamia; es decir, evaluar si la liberación del polen y la receptividad del estigma ocurren simultáneamente en la flor, fue estimada de la siguiente manera: en las flores marcadas se registró el día en el que quedó expuesto el polen. En el primer y cuarto día de vida de las flores, la receptividad del estigma se evaluó al realizar exocruzas en las mismas flores donde se registró la exposición del polen. Dichas flores fueron previamente embolsadas desde que fueron botones florales para evitar contaminación con polen de otra flor (en tres flores / planta / cinco plantas / tratamiento). Las flores se volvieron a cubrir después de realizar los tratamientos de polinización. Se decidió utilizar este método ya que se considera la mejor manera de evaluar la receptividad del estigma, a diferencia del uso de peróxido de hidrógeno que puede ser una prueba un tanto subjetiva (Kearns e Inouye, 1993). Sin embargo, no fue posible realizar las exocruzas durante los cinco días consecutivos que dura en promedio una flor, por lo que las exocruzas se realizaron únicamente en el primero y cuarto día. En todos los casos se utilizó polen de otras plantas de flores que acababan de abrir.

Para evaluar la existencia de polinización espontánea se marcaron diez plantas y en cada una se embolsaron diez botones florales, las bolsas se retiraron hasta que las flores se marchitaron y se registró si hubo producción de frutos. En otras diez plantas con flores embolsadas se realizaron los siguientes tratamientos: (1) Autopolinización manual, en el cual se depositó el polen en el estigma de la misma flor. (2) Exocruzas, las cuales se realizaron durante el cuarto día de vida de las flores, para asegurar que el estigma estuviera receptivo (ver resultados dicogamia), al depositar polen de plantas alejadas al menos por $10 \mathrm{~m}$, posteriormente se volvieron a embolsar hasta la caída de las flores. Debido a que el estigma está exerto (separado de las anteras), y al hecho de que las anteras permanecen ocultas en el labio superior de la corola, no fue necesario emascular las flores usadas en este tratamiento. (3) Finalmente, se marcaron flores con- 
trol, las cuales estuvieron expuestas a los polinizadores. En todos los casos se usaron cinco flores por planta por tratamiento, y la colecta de frutos se realizó aproximadamente cuatro semanas después de haber sido marcadas las flores. Las comparaciones de la proporción de frutos y el número de semillas por fruto, entre los distintos tratamientos, se analizaron por medio de una ANOVA utilizando los promedios por planta como unidad de réplica y posteriormente se realizó una prueba de comparación de medias (Tukey). Las proporciones de frutos fueron transformadas por medio del arcoseno de la raíz cuadrada y el número de semillas por medio del logaritmo natural con la finalidad de ajustar los datos a una distribución normal.

Robo de néctar. En cada una de las tres poblaciones se marcaron diez plantas y, en cada una de ellas se protegió el cáliz
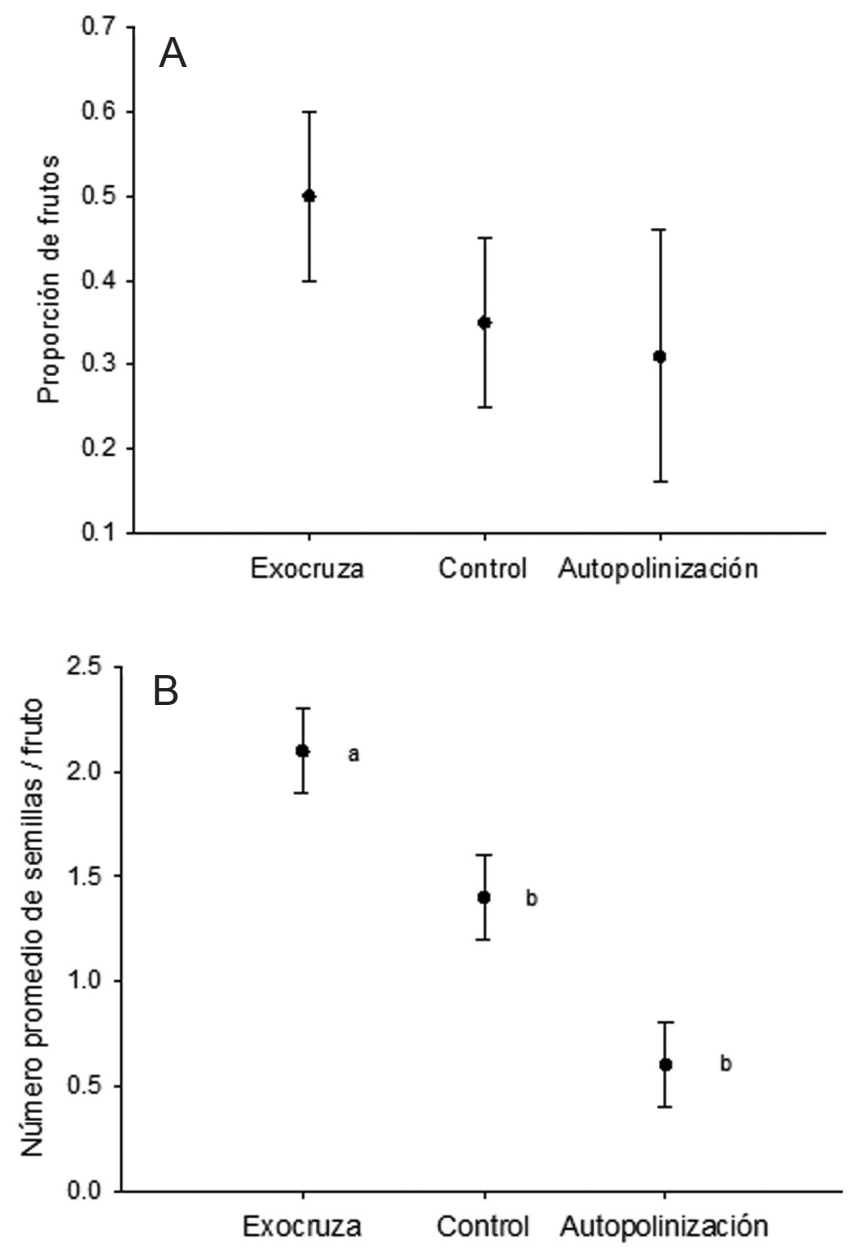

Figura 1. A. Promedios de frutos por tratamiento producto de exocruzas, controles y autofertilizaciones manuales. Los promedios no difieren significativamente. B. Número promedio de semillas obtenidas para los mismos tratamientos. Las flores autofertilizadas manualmente produjeron significativamente menos semillas que las flores de exocruza y controles. Letras distintas muestran diferencias entre tratamientos. de diez botones con un aro de popote transparente, el cual se ha observado que no afecta las visitas de los polinizadores. Otros diez botones de las mismas plantas se marcaron como control y fueron dejados expuestos a los polinizadores y robadores. Las flores fueron visitadas periódicamente hasta que los frutos maduraron y las semillas fueron colectadas. En las tres poblaciones se estimó la proporción de frutos, el número de semillas por fruto y el peso de semillas. Para probar el efecto de la población en la proporción de frutos y el número de semillas por fruto se realizó un análisis de varianza de una vía. En este análisis, el factor fue la población (tres niveles) y la variable de respuesta fue la proporción relativa de frutos o la producción relativa de semillas por fruto. La proporción relativa de frutos $(p f)$ se calculó como:

$$
P_{f}=\frac{p f,-p f_{n r}}{p f_{n r}}
$$

Donde $p f r$ es la proporción de frutos de las flores robadas y pfnr es la proporción de frutos de las flores protegidas de robadores de una planta. Este cociente toma un valor de cero si la proporción de frutos es similar entre flores robadas y no robadas en una misma planta. Es menor de cero si la proporción de frutos es mayor en flores no robadas (indicando un efecto negativo del robo) y es mayor de cero si la proporción de frutos es mayor en flores robadas (indicando un efecto positivo del robo). Una fórmula similar se usó para la producción relativa de semillas por fruto. Las comparaciones al interior de cada población, de la proporción de frutos y el número de semillas por fruto, entre frutos robados y no robados, se realizaron por medio de pruebas pareadas de $t$ de Student usando los promedios por planta como unidad de réplica. Además, en la población tres se estimó la tasa de germinación de semillas provenientes de frutos robados (diez plantas, dos frutos / planta y entre tres y, cinco semillas / planta, total 38 semillas) y no robados (diez plantas, dos frutos / planta y entre tres y, cinco semillas / planta, total 48 semillas). Las semillas se sembraron a $0.5 \mathrm{~cm}$ de profundidad, en tierra comercial, en macetas de $10 \mathrm{~cm}$, que fueron mantenidas en un invernadero a temperatura ambiente en el campus de la Universidad Michoacana en la ciudad de Morelia. La germinación se estimó como la emergencia de los cotiledones y se analizó utilizando una prueba de $t$ de Student, usando a la planta como unidad de réplica.

\section{Resultados}

Biología reproductiva. En todas las flores medidas, el largo del estigma sobrepasó al de las anteras por $2.68 \pm 0.38 \mathrm{~mm}$ (media \pm error estándar; hercogamia de acercamiento). Respecto a la evaluación de la dicogamia, las dos anteras de todas las flores marcadas abrieron simultáneamente el primer día de vida de las flores durante el transcurso de la mañana, 

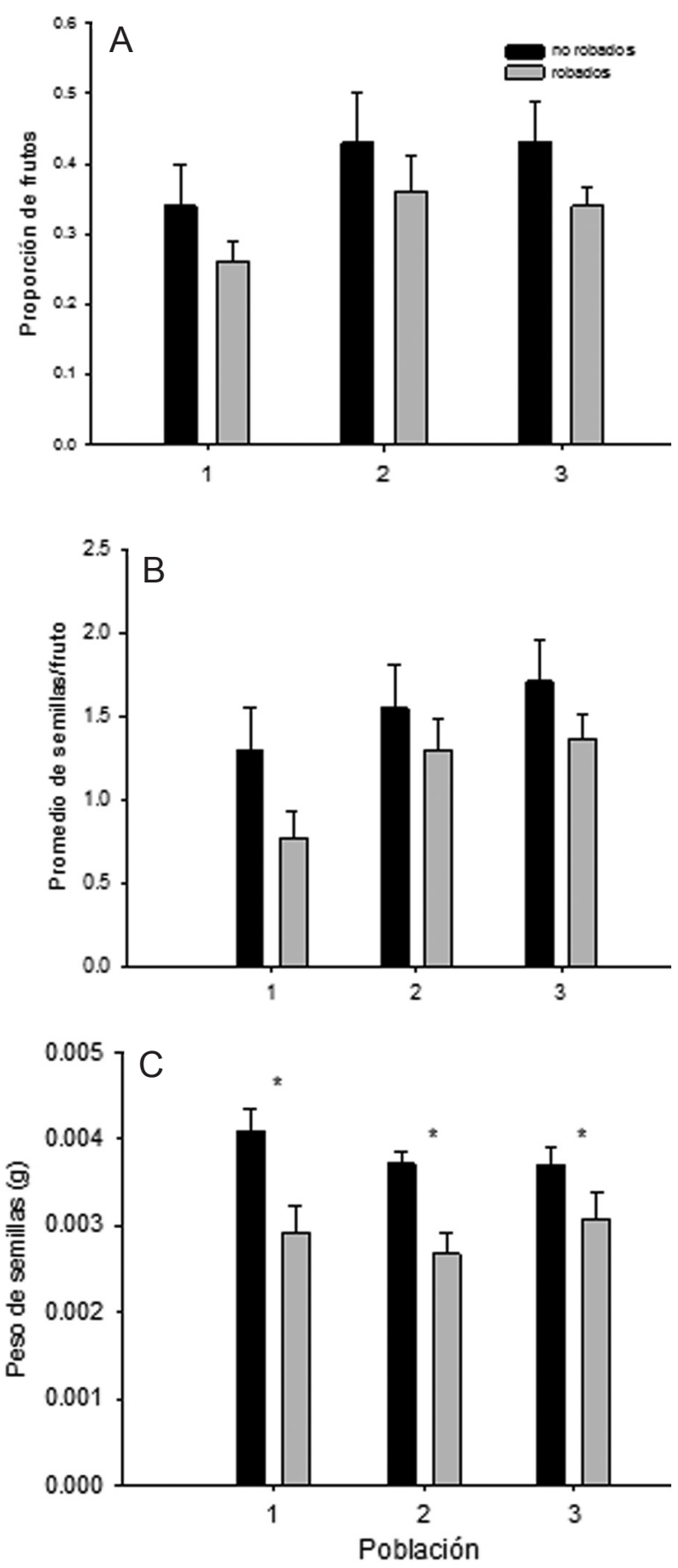

Figura 2. Producción de frutos, semillas y peso de semillas de flores robadas y no robadas (protegidas artificialmente). No se detectaron diferencias en la producción de frutos, al interior de las tres poblaciones estudiadas. A. Pob. 1: $t_{8}=1.11, P=0.29$; Pob. 2: $t_{9}=0.83, P=0.42$; Pob. $3: t_{9}=1.39, P=0.19$; ni en el número de semillas por fruto. B. Pob. 1: $t_{8}=-0.88, P=0.4$; Pob. 2: $t_{9}=-0.36, P=0.72$; Pob. 3: $t_{9}=-1.04, P=0.32$. Únicamente el peso de las semillas no robadas fue significativamente mayor en las tres poblaciones. C. Pob. 1: $t_{8}=-3.04, P<0.01$; Pob. 2: $t_{9}=-3.39, P<0.01$; Pob. $3: t_{9}=-2.5, P=0.03$. El $*$ muestra las diferencias significativas. aunque no fue posible registrar la hora exacta de la dehiscencia. Las exocruzas realizadas durante el primer día de vida de las flores no produjeron semillas viables, probablemente debido a que el estigma no estaba receptivo, mientras que el $66 \pm 8 \%$ de las flores polinizadas al cuarto día pasaron a fruto con un promedio de $3.5 \pm 0.16$ semillas por fruto. Con esta información se puede decir que las flores de Salvia gesneriflora son protándricas; es decir, el polen queda expuesto antes de que el estigma sea receptivo. Sin embargo, es probable que ocurra cierto traslape entre las funciones sexuales en los casos en los que el polen no sea removido el primer o segundo día de vida de las flores. De las 100 flores embolsadas (autofecundación espontánea), únicamente dos produjeron fruto (proporción de frutos $=2 \%$ ). No se detectaron diferencias en la proporción de frutos producidos por autofecundación manual, controles y exocruzas $\left(\mathrm{F}_{2,32}=1.53\right.$, $P=0.23$; Figura 1A). Sin embargo, las flores que se autofecundaron manualmente produjeron $(75 \%)$ menos semillas que las polinizadas por exocruza $\left(\mathrm{F}_{2,21}=14.17, P<0.0001\right.$; Figura 1B).

Robo de néctar. No se detectaron diferencias significativas entre las tres poblaciones en la proporción relativa de frutos (F $=0.07, g l=2,27, P=0.92)$, ni de semillas ( $\mathrm{F}=0.04$, $g l=2,27, P=0.36)$ por fruto. De manera similar, al interior de cada una de las tres poblaciones no se detectaron diferencias significativas en la proporción de frutos (Figura 2A), ni en el número de semillas por fruto (Figura 2B); sólo el peso de semillas de flores no robadas fue mayor en las tres poblaciones (Figura 2C). Finalmente, el promedio del porcentaje de germinación de semillas de flores no robadas $(91.6 \pm 3.72 \%)$ fue significativamente mayor que el de semillas de flores robadas $(62.5 \pm 10.03 \%, t=-2.72, P=0.01)$.

\section{Discusión}

Los resultados de este trabajo apoyan la hipótesis de que el robo de néctar puede tener consecuencias negativas en el éxito reproductivo en especies con sistemas reproductivos principalmente de exocruza que reducen la autofecundación (Claßen-Bockhoff et al., 2004; Westerkamp y ClaßenBockhoff, 2007; Armbruster et al., 2009). Las flores de Salvia gesneriflora son autocompatibles, pero es poco probable que ocurra la polinización espontánea debido a la separación espacial (hercogamia) y temporal (dicogamia) entre las funciones sexuales de sus flores. Sin embargo, el $2 \%$ de las flores embolsadas produjeron fruto; lo cual probablemente se debió a un efecto de las bolsas que protegieron las flores y que pudieron empujar el pistilo hacia las anteras.

Además de la hercogamia y dicogamia, como mecanismos que pueden promover la exocruza, se encontró una disminución del $75 \%$ en la producción de semillas en flores autofecundadas manualmente, que probablemente sea la expresión de la depresión endogámica (Charlesworth y Char- 
lesworth, 1987), lo que concuerda con que Salvia gesneriflora es una especie principalmente de exocruza (Husband y Schemske, 1996). Otra posibilidad es que la disminución de semillas se deba a un mecanismo de auto-incompatibilidad parcial. Sin embargo, debido a que no se han reportado sistemas de incompatibilidad en el género Salvia (Haque y Ghoshal, 1981), se considera que esta posibilidad es poco probable. Es recomendable evaluar el efecto de la endogamia en más componentes del ciclo de vida (p. ej. porcentaje de germinación, supervivencia y fecundidad), y estimar la tasa de autofecundación para conocer la relación del sistema de cruza y la evolución de la endogamia en esta especie.

A pesar de que Salvia gesneriflora es autocompatible, la hercogamia y dicogamia de sus flores disminuye la posibilidad de que los robadores contribuyan a la autopolinización durante el robo de néctar, como se ha reportado en otras especies (Navarro, 2000; Zhang et al., 2009). El robo de néctar afectó el peso de semillas y su tasa de germinación, componentes relevantes del éxito reproductivo; mientras que la producción de frutos y semillas no disminuyó de manera significativa, similar a lo que se ha reportado en otra especie de Salvia (Arizmendi et al., 1996). Además, el efecto del robo fue similar en las tres poblaciones, lo que demuestra que tanto la presión de los robadores como la respuesta de $S$. gesneriflora fueron homogéneas en el área de distribución estudiada. La relación entre el robo de néctar y la disminución del peso de las semillas probablemente se deba a que durante el robo se daña el cáliz, el cual puede ayudar en la maduración de las semillas (Herrera, 2005). Por lo tanto, al ser perforado el cáliz por los robadores, los óvulos podrían no completar un desarrollo óptimo, lo que produce semillas más pequeñas con una menor tasa de germinación. Otra posibilidad es que al perforar las flores, los robadores estimulen la generación de néctar y comprometan los recursos que la planta puede asignar al desarrollo de las semillas (Roubik et al., 1985; Pyke, 1991; Navarro, 1999). Sin embargo, la estimulación de la producción de néctar también puede ser ocasionada por las visitas de los colibríes.

Por otra parte, aunque en este estudio no se evaluaron los daños que ocasionan distintos robadores, ni si estos disminuyen la calidad de las semillas (cercanía del daño con los óvulos), observaciones preliminares muestran que el robo por aves tiene un efecto negativo mayor en distintos componentes del éxito reproductivo de Salvia gesneriflora que el robo por insectos (Baños-Bravo, 2010)

Se puede concluir que el robo de néctar afectó negativamente el peso de las semillas de Salvia gesneriflora y su tasa de germinación. Es posible que con tamaños de muestra mayores se detecte un efecto del robo de néctar en la producción de frutos y semillas. Además, la presencia de hercogamia y dicogamia sugiere que es una especie que favorece la exocruza, por lo que los robadores no parecen contribuir a la polinización como en otras especies.

\section{Agradecimientos}

A la Coordinación de la Investigación Científica de la UMS$\mathrm{NH}$ por haber apoyado la realización de este estudio y a dos revisores anónimos por sus valiosas contribuciones, las cuales mejoraron sustancialmente este trabajo.

\section{Literatura citada}

Alcalá-Guerra A. 2011. Biología reproductiva y fenología floral de un arbusto de bosque templado. Tesis de licenciatura, Facultad de Biología, Universidad Michoacana de San Nicolás de Hidalgo, Morelia, Michoacán, México. 34 pp.

Arizmendi M.C., Domínguez C.A. y Dirzo R. 1996. The role of an avian nectar robber and of hummingbird pollinators in the reproduction of two plant species. Functional Ecology 10:119-127.

Armbruster W.S., Hansen T.F., Pélabon C., Pérez-Barrales R. y Maad J. 2009. The adaptive accuracy of flowers: measurement and microevolutionary patterns. Annals of Botany 103:15291545.

Baños-Bravo Y.E. 2010. Frecuencia del robo de néctar y sus efectos en la adecuación masculina de un arbusto de bosque templado. Tesis de licenciatura, Facultad de Biología, Universidad Michoacana de San Nicolás de Hidalgo, Morelia, Michoacán, México. 45 pp.

Carranza-González E. 1987. Aspectos botánico-ecológicos del campo geotérmico Los Azufres, Michoacán, México. Tesis de licenciatura, Facultad de Biología, Universidad Michoacana de San Nicolás de Hidalgo, Morelia, Michoacán, México. 59 pp.

Charlesworth D. y Charlesworth B. 1987. Inbreeding depression and its evolutionary consequences. Annual Review of Ecology and Systematics 18:237-268.

Claßen-Bockhoff R., Wester P. y Tweraser E. 2004. The staminal lever mechanism in Salvia L. (Lamiaceae) - A review. Plant Biology 5:33-41.

Haque M.S. y Ghoshal K.K. 1981. Floral biology and breeding system in the genus Salvia L. Proceedings of the Indian National Science Academy B47 5:716-724.

Heil M. 2011. Nectar: generation, regulation and ecological functions. Trends in Plant Science 16:191-200.

Herrera C.M. 2005. Post-floral perianth functionaliy: contribution of persistent sepals to seed development in Helleborus foetidus (Ranunculaceae). American Journal of Botany 92:1486-1491.

Husband B.C. y Schemske D.W. 1996. Evolution of the magnitude and timing of inbreeding depression in plants. Evolution 50:54-70.

Inouye D.W. 1980. The terminology of floral larceny. Ecology 61:1251-1253.

Irwin R.E. 2000. Hummingbird avoidance of nectar-robbed plants: spatial location or visual cues. Oikos 91:499-506.

Irwin R.E. y Brody A.K. 1998. Nectar robbing in Ipomopsis aggregata: effects on pollinator behavior and plant fitness. Oecologia 116:519-527.

Irwin R.E, Brody A.K. y Waser N.M. 2001. The impact of floral larceny on individuals, populations, and communities. Oecologia 129:161-168.

Irwin R.E., Bronstein J.L., Manson. J.S. y Richardson L. 2010. Nectar robbing: Ecological and evolutionary perspectives. Annual Review of Ecology and Systematics 41:271-292. 
Kearns C.A. e Inouye D.W. 1993. Techniques for Pollination Biologists. University Press of Colorado, Niwot.

Maloof J.E. e Inouye D.W. 2000. Are nectar robbers cheaters or mutualists? Ecology 81:2651-661.

Navarro L. 1999. Pollination ecology and effect of nectar removal in Macleania bullata (Ericaceae). Biotropica 31:618-625.

Navarro L. 2000. Pollination ecology of Anthyllis vulneraria subsp. vulgaris (Fabaceae): Nectar robbers as pollinators. American Journal of Botany 87:980-985.

Ornelas J.F., Ordano M., De Nova A.J., Quintero M.E. y Garland T. 2007. Phylogenetic analysis of interspecific variation in nectar of hummingbird-visited plants. Journal of Evolutionary Biology 20:1904-1917.

Pyke G.H. 1991. What does it cost a plant to produce floral nectar? Nature 350:58-59.

Ramamoorthy T.P. 2005. Salvia L. En: Calderón de Rzedowski G. y Rzedowski J. Eds. Flora Fanerogámica del Valle de México, pp. 632-643, Comisión Nacional para el Conocimiento y Uso de la Biodiversidad,- Instituto de Ecología, A.C., México, D.F.

Real L. 1983. Introduction. En: Real L. Ed. Pollination Biology, pp. 1-5, Academic Press, Nueva York.

Roubik D.W., Holbrook N.M. y Parra G.V. 1985. Roles of nectar robbers in reproduction of the tropical treelet Quassia amara (Simaroubaceae). Oecologia 66:161-167.

Wester P. y Claßen-Bockhoff R. 2007. Floral diversity and pollen transfer mechanisms in bird-pollinated Salvia species. Annals of Botany 100:401-421.

Westerkamp C. y Claßen-Bockhoff R. 2007. Bilabiate flowers: The ultimate response to bees? Annals of Botany 100:361-374.

Zhang Y., Yu Q., Zhao J. y Guo Y. 2009. Differential effects of nectar robbing by the same bumble-bee species on three sympatric Corydalis species with varied mating systems. Annals of Botany 104:33-39.

Recibido: 12 de octubre de 2012

Aceptado: 7 de enero de 2013 